\title{
Hyponatremia as predictor of worse outcome in real world patients admitted with acute heart failure
}

Jose Carlos Arévalo Lorido ${ }^{1}$, Juana Carretero Gómez ${ }^{1}$, Francesc Formiga ${ }^{2}$,

Manuel Montero Pérez-Barquero ${ }^{3}$, Joan Carles Trullás Vila ${ }^{4}$, Oscar Aramburu Bodas ${ }^{5}$, Luis Miguel Ceresuela ${ }^{6}$; the RICA Investigators*

${ }^{1}$ Internal Medicine Service, Hospital de Zafra, Zafra, Badajoz, Spain

${ }^{2}$ Internal Medicine Service, Hospital University de Bellvitge, L'Hospitalet del Llobregat, Barcelona, Spain ${ }^{3}$ Internal Medicine Service, Hospital University Reina Sofía, Córdoba, Spain

${ }^{4}$ Internal Medicine Service, Hospital Sant Jaume de Olot, Girona, Spain

${ }^{5}$ Internal Medicine Service, Hospital Virgen de la Macarena, Sevilla, Spain

${ }^{6}$ Internal Medicine Service, Hospital General de L'Hospitalet, L'Hospitalet del Llobregat, Barcelona, Spain

\begin{abstract}
Background: Our aim was to determine if hyponatremia, defined as serum sodium level $<135 \mathrm{mmol} / \mathrm{L}$, is a predictor of worse outcome in a cohort of real-world patients with heart failure $(H F)$.

Methods: We used data of the National registry of HF (RICA) from Spain, an ongoing multicenter, prospective cohort study. The patients were assigned to two groups regarding sodium levels. Primary end-point was first all-cause readmission, or death by any cause. Secondary end-points were the number of days hospitalized, and the presence of complications.

Results: We identified 973 patients, 147 (15.11\%) with hyponatremia. The median age of patients enrolled was $77.25 \pm 8.79$ years-old, the global comorbidity measured by Charlson comorbidity index (CCI) was upper 3 points and preserved ejection fraction was present in $67.1 \%$ of them. Clinical complications during admission were significantly higher in the patients with hyponatremia $(35.41 \%, p<0.001)$ and this remained as significant predictor after logistic regression adjustment $(O R$ 1.08, $p<0.01)$. Also mortality and readmissions were more frequent in patients with hyponatremia (20.69\% and $22.41 \%$, respectively) but after Cox regression adjustment hyponatremia in our cohort was not associated with increase in 90-day all-cause mortality and readmissions, and only CCI remained significant for primary end-point (HR 1.08, $p<0.001)$.

Conclusions: Hyponatremia is an independent predictor of complications during hospitalization in our real-world cohort, but was not associated with 90 days mortality or readmissions. Global comorbidity, however, played an important role, and could influence the mortality and readmissions of our patients. (Cardiol J 2013; 20, 5: 506-512)
\end{abstract}

Key words: heart failure, hyponatremia, sodium

Address for correspondence: José Carlos Arévalo Lorido. Internal Medicine Service. Hospital de Zafra, Ctra Badajoz-Granada 432. Zip Code: 06300, Zafra (Badajoz), Spain, tel: 0034+924029200, e-mail: joscarlor@gmail.com

*The RICA Investigators are listed in the Appendix 1.

Received: 26.11.2012 Accepted: 05.02.2013 


\section{Introduction}

Hyponatremia defined as serum sodium level $<135 \mathrm{mmol} / \mathrm{L}$, is a common electrolyte abnormality in hospitalized patients, and it is associated with increasing morbidity and mortality.

Patients with heart failure (HF) are not exempt from this association, and $19 \%$ to $25 \%$ of patients with acute decompensated heart failure (ADHF) have hyponatremia $[1,2]$.

In the last few years, several studies reported that plasma sodium level on admission is an independent predictor of increased rates of rehospitalization and mortality in patients hospitalized for $\mathrm{HF}$ [3-5], it is also associated with longer length of stay [1], and is more likely to require dialysis and inotropic agents [1]. However, the question whether there is a cause-and-effect mechanism remains unclear.

In most of these studies, patients were admitted in Cardiology wards. They had several differences with the patients usually admitted to internal medicine wards regarding the age, comorbidity and etiology of HF. In addition, most of these studies had not been designed to investigate exclusively the predictive value of hyponatremia in the clinical course of patients with HF.

Our aim was to determine the relationship between hyponatremia and prognosis in patients with $\mathrm{HF}$ admitted to internal medicine departments.

\section{Methods}

Patients were recruited through the National Registry of Heart Failure (RICA), supported by the Heart Failure-Working Group of the Spanish Society of Internal Medicine (SEMI-IC). The RICA Registry is an ongoing multicenter, prospective, cohort study that has been described elsewhere $[6,7]$. This registry included consecutive and unique patients with $\mathrm{HF}$ according to the criteria of the European Society of Cardiology [8]. The patients were exclusively admitted to internal medicine wards of 52 public and private hospitals in Spain between March 2008 and October 2011. They were included on discharge after an $\mathrm{ADHF}$, and then they were followed-up for 1 year, with visits at 3 and 12 months after inclusion.

The study protocol was approved by the Ethics Committee of the University Hospital "Reina Sofia”, Córdoba (Spain), and all patients provided their informed consent. Data were collected through a web site (https://www.registrorica.org), which contained the database, accessed with a personal password. Confidentiality was preserved since no personal data was stored, except date of birth and name initials to avoid data duplication.

\section{Data collection and follow-up}

Upon admission we obtained a comprehensive medical history and undertook a detailed physical examination. We assessed comorbidity with Charlson index (CCI) [9], basal functional status with Barthel index [10], and cognitive status with Pfeiffer questionnaire [11]. We also registered data of laboratory evaluation including levels of creatinine, urea, glucose, sodium, potassium and hemoglobin, complications during admission and prescriptions at discharge. We excluded patients whose laboratory data were not fully completed, patients without echocardiographic examination and patients who died during hospitalization. All patients in this study were followed for 90 days after discharge. For rehospitalized patients or those who died during follow-up, data on the cause of readmission or death were obtained.

Hyponatremia was defined as levels of serum sodium under $135 \mathrm{mmol} / \mathrm{L}$ and all patients were hypervolemic. Each patient was assigned to one of the following two groups: hyponatremia if sodium $<135 \mathrm{mmol} / \mathrm{L}$, and normonatremia if sodium $\geq 135 \mathrm{mmol} / \mathrm{L}$. The patients with levels of serum sodium $\geq 148 \mathrm{mmol} / \mathrm{L}$, were also excluded.

\section{End-points}

The primary end-point was either first all-cause readmission, or death by any cause. Secondary end-points were the number of days hospitalized, and the presence of complications, such as worsening of renal function (defined by elevation of creatinine basal levels $\geq 2 \mathrm{mg} / \mathrm{dL}$ ), hyperpotasemia (defined by elevation of serum potassium $\geq 5.5 \mathrm{mg} / \mathrm{dL}$ ), acute confusional syndrome, urinary tract infections, pneumonia, and needing for blood transfusion, hemofiltration or needing for either inotropic or hypotensive drugs.

\section{Statistical analysis}

We compared variables between patients with hyponatremia or normal sodium levels, who were used as the reference group. We firstly conducted univariable analysis between the groups. We used the $\chi^{2}$ test for dichotomous and qualitative variables, and the Kruskal-Wallis test for continuous variables. We then conducted multivariable analysis by Cox regression model for morbidity and mortality outcome variables, adjusted to sig- 
Table 1. Baseline characteristics of the patients with or without hyponatremia. Values are given as number $(\%)$ or as median (interquartile range).

\begin{tabular}{|c|c|c|c|}
\hline Variable & $\mathrm{Na}<135 \mathrm{mmol} / \mathrm{L}$ & $\mathrm{Na} \geq 135 \mathrm{mmol} / \mathrm{L}$ & $\mathbf{P}$ \\
\hline $\mathrm{N}$ & 147 & 826 & \\
\hline Age [years] & $78(12 \%)$ & $79(10 \%)$ & 0.85 \\
\hline Gender (men) & $64(43.54 \%)$ & $386(46.73 \%)$ & 0.47 \\
\hline Hypertension & $125(85.03 \%)$ & $688(83.29 \%)$ & 0.59 \\
\hline Diabetes & $74(50.34 \%)$ & $339(43.46 \%)$ & 0.12 \\
\hline Alcohol & $22(14.97 \%)$ & $197(12.83 \%)$ & 0.15 \\
\hline Smoking & $14(9.52 \%)$ & $67(8.11 \%)$ & 0.56 \\
\hline Dyslipidemia & $72(48.98 \%)$ & $362(43.83 \%)$ & 0.24 \\
\hline Myocardial infarction & $37(25.17 \%)$ & $165(19.98 \%)$ & 0.57 \\
\hline Cerebrovascular disease & $19(12.93 \%)$ & $107(12.95 \%)$ & 0.94 \\
\hline Peripheral vascular disease & $16(10.88 \%)$ & $109(13.20 \%)$ & 0.87 \\
\hline Renal disease & $71(48.3 \%)$ & $281(34.02 \%)$ & 0.005 \\
\hline $\mathrm{CCl}$ & $4(4 \%)$ & $3(4 \%)$ & 0.001 \\
\hline Barthel index & $90(30 \%)$ & $95(25 \%)$ & 0.17 \\
\hline Pfeiffer index & $1(3 \%)$ & $1(3 \%)$ & 0.16 \\
\hline \multicolumn{4}{|l|}{ CHF severity (NYHA functional class) } \\
\hline I & $9(6.12 \%)$ & $77(9.32 \%)$ & 0.42 \\
\hline II & $67(45.58 \%)$ & $396(47.94 \%)$ & 0.42 \\
\hline III & $61(41.5 \%)$ & $711(37.65 \%)$ & 0.42 \\
\hline IV & $10(6.8 \%)$ & $42(5.08 \%)$ & 0.42 \\
\hline Heart rate [bpm] & $82(28 \%)$ & $86(31 \%)$ & 0.21 \\
\hline SBP $[\mathrm{mm} \mathrm{Hg}]$ & $130(42 \%)$ & $140(39 \%)$ & 0.002 \\
\hline DBP [mm Hg] & $72(18 \%)$ & $78(24 \%)$ & 0.002 \\
\hline Creatinine [mg/dL] & $1.32(0.8 \%)$ & $1.14(0.6 \%)$ & 0.004 \\
\hline Hemoglobin [g/dL] & $12.1(2.4 \%)$ & $12.1(3 \%)$ & 0.08 \\
\hline Glucose [mg/dL] & $122(87 \%)$ & $113(56 \%)$ & 0.009 \\
\hline Urea [mg/dL] & $66(60 \%)$ & $57(36 \%)$ & 0.006 \\
\hline Potassium [mmol/L] & $4.4(0.8 \%)$ & $4.2(0.7 \%)$ & 0.006 \\
\hline EF & $50(20 \%)$ & $53(23 \%)$ & 0.19 \\
\hline Preserved EF & $99(67.35 \%)$ & $553(66.95 \%)$ & 0.92 \\
\hline Clinical complications during admission & $53(36.05 \%)$ & $186(22.52 \%)$ & 0.0004 \\
\hline Pleural effussion & $68(46.26 \%)$ & $289(34.99 \%)$ & 0.009 \\
\hline Atrial flutter/fibrillation & $84(57.14 \%)$ & $435(52.66 \%)$ & 0.26 \\
\hline Pro-BNP [60/303] & $3879.5(8322.5 \%)$ & $3043(5652.0 \%)$ & 0.56 \\
\hline
\end{tabular}

$\mathrm{CCl}$ - Charlson comorbidity index; CHF — chronic heart failure severity; SBP — systolic blood pressure; DBP - diastolic blood pressure; $\mathrm{EF}$ - ejection fraction; Pro-BNP — B type natriuretic peptide; obtained in 60 patients with hyponatremia and 303 patients with normal serum sodium

nificant variables in univariable analysis (outcome were defined as the time to the event or at least 90 days of follow-up if an event did not occur). A logistic regression model was used to analyze the complications at admission adjusted to variables with significant differences in univariable analysis.

Cumulative event curves were estimated by Kaplan-Meier method and compared by Cox regression test. Differences and correlations were considered significant at $\mathrm{p}<0.05$.

\section{Results}

We enrolled 973 patients $(46.25 \%$ men) admitted for ADHF with a mean of $77.2 \pm 8.8$ years. Hyponatremia was present in 147 (15.1\%) patients. Nobody had severe hyponatremia (serum sodium $\leq 120 \mathrm{mmol} / \mathrm{L}$ ). Baseline characteristics of the patients are showed in Table 1 . The patients with hyponatremia had significantly more global comorbidity measured by CCI and more frequency of renal failure. Levels of glucose, urea, creatinine, 
Table 2. Clinical complications in heart failure patients with and without hyponatremia. Values are given as number $(\%)$ or as median (interquartile range).

\begin{tabular}{|c|c|c|c|}
\hline Variable & $\begin{array}{c}\mathrm{Na}<135 \mathrm{mmol} / \mathrm{L} \\
(\mathrm{n}=147)\end{array}$ & $\begin{array}{c}\mathrm{Na} \geq 135 \mathrm{mmol} / \mathrm{L} \\
(\mathrm{n}=826)\end{array}$ & $\mathbf{P}$ \\
\hline Length of stay hospitalized & $9(8 \%)$ & $7.5(7 \%)$ & 0.0005 \\
\hline Total complications & $53(36.05 \%)$ & $186(22.52 \%)$ & 0.0004 \\
\hline Worsening of renal function & $22(14.97 \%)$ & $68(8.23 \%)$ & 0.009 \\
\hline Hyperpotassemia & $12(8.16 \%)$ & $39(4.72 \%)$ & 0.08 \\
\hline Need for vasoactive drugs & $9(6.12 \%)$ & $22(2.66 \%)$ & 0.02 \\
\hline Need for hypotensive drugs & $22(14.97 \%)$ & $62(7.51 \%)$ & 0.003 \\
\hline Need for ultrafiltration & $1(0.68 \%)$ & $0(0 \%)$ & 0.017 \\
\hline Need for blood & $6(4.08 \%)$ & $31(3.75 \%)$ & 0.84 \\
\hline Acute confusional status & $20(13.61 \%)$ & $46(5.57 \%)$ & 0.0004 \\
\hline Pneumonia & $3(2.04 \%)$ & $12(1.45 \%)$ & 0.59 \\
\hline Urinary infection & $12(8.16 \%)$ & $29(3.51 \%)$ & 0.009 \\
\hline Tromboembolic disease & $1(0.68 \%)$ & $6(0.73 \%)$ & 0.95 \\
\hline
\end{tabular}

and potassium were significantly higher in the group of hyponatremia, and blood pressure was significantly lower.

The patients (both normonatremics and hyponatremics) taking either angiotensin converting enzyme inhibitors or angiotensin receptor blokers were $76.7 \%$, loops diuretics $81.2 \%$, thiazides $10.9 \%$ and $53.4 \%$ were receiving beta-blockers. There were no differences between the two groups of patients.

Clinical complications and pleural effusion during admission were higher in the group of patients with hyponatremia $(35.41 \%, \mathrm{p}<0.001)$. Findings are shown in Table 2 . This group suffered more worsening of basal renal function, more hyperpotasemia, more need for vasoactive and hypotensive drugs, more need for hemofiltration, more frequency of acute confusional status, and more frequency of urinary infections than the group with normal sodium. Hyponatremia (OR 1.63, $\mathrm{p}<0.01)$ and CCI (OR 1.08, $\mathrm{p}<0.01)$ remained as significant independent predictors when entered into the adjusted logistic regression model as shown in Table 3.

From the initial sample, 763 patients, 647 with normal sodium and 116 with hyponatremia completed the follow-up (11 patients were lost and 199 had not arrived to 90-day visit yet). Mortality occurred in $73(11.4 \%)$ patients with normal sodium and in $24(20.7 \%)$ with hyponatremia $(\mathrm{p}<0.006)$. Readmission was also more frequent in patients with hyponatremia (Table 4). However, by adjusting the primary end-point through Cox regression test, hyponatremia was not associated neither
Table 3. Factors associated with clinical complications in logistic regression model, adjusted for significant variables in univariable analysis.

\begin{tabular}{lccc}
\hline Variable & Odd ratio & $95 \% \mathbf{C l}$ & $\mathbf{P}$ \\
\hline SBP & 1.00 & $0.99-1.00$ & 0.97 \\
DBP & 1.00 & $0.99-1.01$ & 0.38 \\
Renal failure & 1.03 & $0.85-1.25$ & 0.74 \\
CCI & 1.08 & $1.01-1.15$ & 0.01 \\
Urea & 1.00 & $0.99-1.00$ & 0.06 \\
Creatinine & 1.15 & $0.86-1.53$ & 0.32 \\
Potassium & 1.14 & $0.89-1.46$ & 0.27 \\
Glucose & 1.00 & $0.99-1.00$ & 0.55 \\
Pleural effusion & 1.24 & $0.91-1.7$ & 0.16 \\
Hyponatremia & 1.63 & $1.10-2.42$ & 0.01 \\
\hline
\end{tabular}

$\mathrm{Cl}$ - confidence interval; SBP - systolic blood pressure; $\mathrm{DBP}$ — diastolic blood pressure; $\mathrm{CCl}$ - Charlson comorbidity index

with mortality nor readmission and only the CCI and systolic blood pressure were (OR 1.08, p $<0.001$, Table 5, Fig. 1).

\section{Discussion}

The main findings that our study showed, were that hyponatremia in HF patients was associated with more clinical complications during admission, but neither with short-term mortality nor its readmissions.

In our cohort, hyponatremia was present in $15.1 \%$ of patients with ADHF. This percentage was slightly lower than frequencies reported in other studies, which ranged between $20 \%$ to $25 \%[1,12$, 13 ] possibly because we did not include the patients 
Table 4. Association of serum sodium levels with outcomes. Univariate analysis was performed using $\chi^{2}$ test and multivariate using Cox regression model adjusted for renal disease, Charlson comorbidity index, systolic and diastolic blood pressure, creatinine, potassium, glucose and urea.

\begin{tabular}{|c|c|c|c|c|c|c|c|c|}
\hline \multirow[t]{2}{*}{ Outcome } & \multicolumn{5}{|c|}{ Univariate analysis } & \multicolumn{3}{|c|}{ Multivariate analysis } \\
\hline & $\begin{array}{c}\mathrm{Na}<135 \mathrm{mmol} / \mathrm{L} \\
(\mathrm{n}=116)\end{array}$ & $\begin{array}{c}\mathrm{Na} \geq 135 \mathrm{mmol} / \mathrm{L} \\
(\mathrm{n}=647)\end{array}$ & HR & $95 \% \mathrm{Cl}$ & $\mathbf{P}$ & HR & $95 \% \mathrm{Cl}$ & $\mathbf{P}$ \\
\hline Readmission & $26(22.41 \%)$ & $113(17.47 \%)$ & 1.36 & $0.84-2.27$ & 0.2 & 1.03 & $0.65-1.64$ & 0.87 \\
\hline Mortality & $24(20.69 \%)$ & 73 (11.44\%) & 2.05 & $1.23-3.41$ & 0.006 & 1.28 & $0.78-2.11$ & 0.3 \\
\hline Primary end-point & $46(39.66 \%)$ & $180(27.82 \%)$ & 1.70 & $1.13-2.56$ & 0.005 & 1.26 & $0.89-1.78$ & 0.18 \\
\hline
\end{tabular}

$\mathrm{HR}$ - hazard ratio; $\mathrm{Cl}$ - confidence interval

Table 5. Factors associated with primary end-point. Cox regression analysis adjusted to significant variables in univariate analysis.

\begin{tabular}{lccc}
\hline Variable & HR & $95 \% \mathbf{C l}$ & P \\
\hline SBP & 0.99 & $0.87-0.99$ & 0.03 \\
DBP & 0.99 & $0.99-1.00$ & 0.87 \\
Renal failure & 1.07 & $0.9-1.27$ & 0.4 \\
CCI & 1.08 & $1.03-1.14$ & 0.001 \\
BUN & 1.00 & $0.99-1.00$ & 0.17 \\
Creatinine & 0.93 & $0.71-1.22$ & 0.62 \\
Glucose & 1.00 & $0.99-1.00$ & 0.56 \\
Hyponatremia & 1.13 & $0.66-1.91$ & 0.64 \\
\hline
\end{tabular}

$\mathrm{HR}$ - hazard ratio; $\mathrm{Cl}$ - confidence interval; SBP - systolic blood pressure; DBP — diastolic blood pressure; BUN — blood urea nitrogen; $\mathrm{CCl}-$ Charlson comorbidity index

who died during hospitalization. Low serum sodium was associated with more clinical complications during admission such as acute confusional status, worsening of renal function, need for vasoactive and hypotensive drugs, and with a higher length of stay, similar to previous studies [1], but also with more urinary infections. This finding has not been not previously reported and may be related to a wider use of urinary catheter for more strict control of diuresis in hyponatremic patients, however, the urinary catheter is widely used in patients hospitalized for any cause, especially in patients with $\mathrm{HF}$, therefore, more studies are necessary to confirm it. Mortality and readmissions were higher in patients with hyponatremia $(20.7 \%$ vs. $11.4 \%$ and $22.4 \%$ vs. $17.5 \%$, respectively). The differences are clinically relevant although there was no association in multivariate analysis.

Our sample includes a real-world cohort of internal medicine patients with ADHF, and dif -fers from others in age (our patients were older with a median of 78-79-year-old), comorbiditiy (CCI higher than 3 points), and in a raised percen-
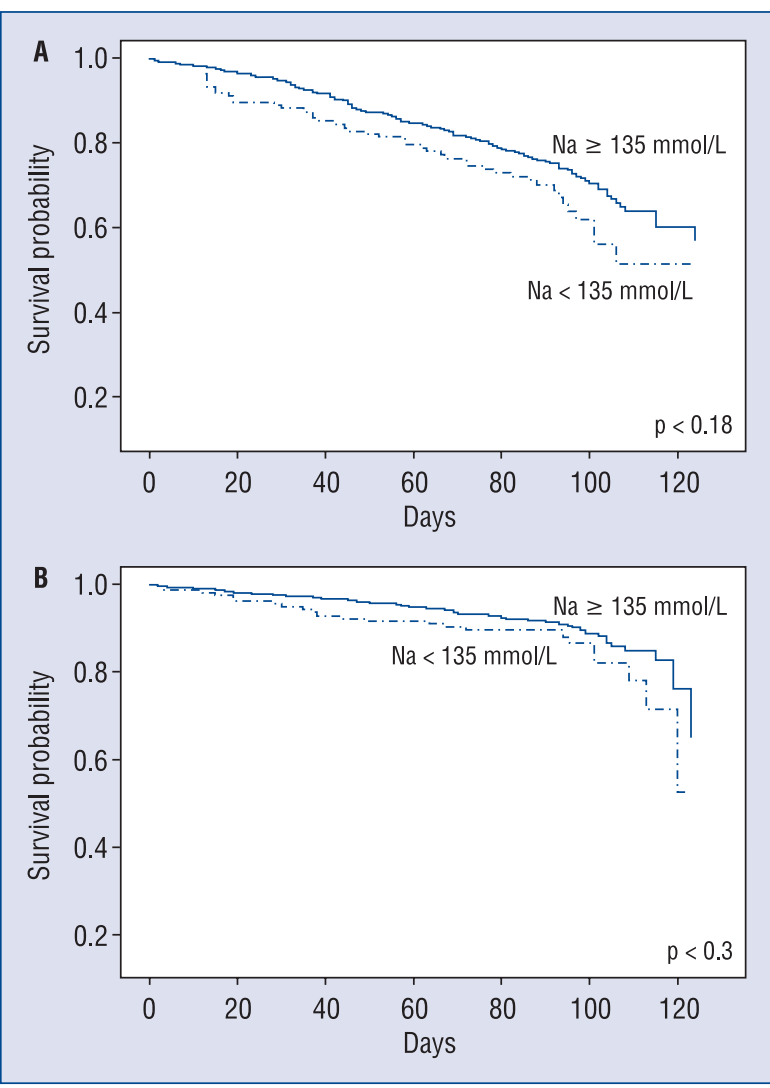

Figure 1. Kaplan Meier survival curves regarding plasma sodium levels. Adjusted for renal disease, Charlson comorbidity index, systolic and diastolic blood pressure, creatinine, potassium, glucose and urea. A. The association with primary end-point; $\mathbf{B}$. The association with all-causes mortality.

tage of patients with non left ventricular systolic dimension ( $67.35 \%$ and $66.95 \%$ by group). Perhaps some of these differences might contribute to the higher mortality shown in this study.

Evidence suggests that the patients who have $\mathrm{HF}$ with preserved ejection fraction, are more likely to be older and to be female [14]. Older 
patients have a higher prevalence of comorbid illnesses than their younger counterparts, and in the setting of $\mathrm{HF}$, age or sex differences have been demonstrated in left ventricular adaptation and autonomic responses, as well as in the clinical course and outcomes of the disease $[15,16]$.

The median age of patients enrolled in previous trials that researched the relationship between serum sodium in patients with $\mathrm{HF}$ and their prognostic was lower $[1,12,13]$ and comorbidity measured by CCI was not included at all, although a recent study shows an increased comorbidity among patients with hyponatremia and HF [17]. In our sample, comorbidity was significantly higher in the patients with hyponatremia and was regarded as both significant and independent variable with clinical complications during admission and with a worse outcome (mortality and readmissions) at 90 days of follow-up (Table 5).

There are other studies that have shown that chronic comorbidities independently increase the mortality rates of patients with newly diagnosed HF [18] and also in ambulatory HF population [19]. In addition, there are other studies that showed a relationship between hyponatremia and a higher comorbidity measured by CCI [20]. In our study, in fact, CCI was higher in the hyponatremic group.

The association between underlying medical comorbidities measured by CCI and mortality in a variety of patients have been demonstrated in several studies [9, 21, 22], however, there are only few of them that use it in the HF population. It could be postulated that there is not a true cause-and-effect between $\mathrm{HF}$ and hyponatremia, but a surrogate marker of comorbidity of these patients. Further studies will be needed to clarify this aspect.

Finally, it has been shown how systolic blood pressure plays a protective role for the primary end-point in our sample (Table 5). Low blood pressure is often associated with hyponatremia in patients with HF [23, 24], and then associated with a worse prognosis in these patients [7], which again suggests that hyponatremia would be a surrogate marker of more severe HF.

Definitely, hyponatremia has shown to be a prognostic indicator of increased mortality in a determined group of patients with HF, as clinical trials were focusing on a relatively small segment of the HF population, however, in wider population, like ours, with older people who have more comorbidity, and more percentage of patients with preserved ejection fraction, this question remains to be investigated, likewise, future trials with hyponatremic patients and $\mathrm{HF}$ should include data of comorbidity to validate the results that we have obtained in this study.

\section{Limitations of the study}

Our present study has several limitations that must be considered. First, the number of patients included was lower than in other studies. In addition, all physicians are enrolled in RICA registry voluntarily, hence our cohort was obtained from patients admitted under the care of a group of specially committed physicians, particularly interested in HF, which could represent a sample bias. Finally, only admission serum sodium levels were considered, so differentials effects of persistent vs. corrected hyponatremia could not be determined. In addition, admission diuretic dose was not collected, consequently, potential association between diuretic dose and hyponatremia could not be examined.

\section{Conclusions}

In conclusion, in this study our real world sample has shown that hyponatremia is a predictor of complications while admission in HF patients, but it has also shown that comorbidity could play an important role in the presence or absence of hyponatremia and could even influence the 90 days mortality or admissions attributed to lower serum sodium. Furthermore, the relationship among both age and comorbidity and their association to survival in HF patients are likely more involved than previously described and demand validation in other studies that include a wide population.

\section{Acknowledgements}

All the investigators from RICA registry. This project was made possible by an unrestricted educational grant from Menarini Laboratories. Our thanks to the Registry Coordinating Center RICA "S \& H Medical Science Service” for its work in quality control of data, logistical and administrative support.

\section{Appendix 1}

Membership of RICA registry: O. Aramburu, J.C. Arévalo, J.L. Arias-Jiménez, L. Anarte, F.J. Carrasco, M. Carrera, J. Casado, L.M. Ceresuela, J.M. Cerqueiro, D. Chivite, A. Conde, J. Díez-Manglano, F. Epelde, F. Formiga, J. Grau, L. Manzano, M. Montero, A. Muela, C. Pérez-Bocanegra, J.I. Pérez-Calvo, B. Prado, J. Recio, C. Sánchez-Sánchez, J.C. Trullàs, A. Urrutia

Conflict of interest: none declared 


\section{References}

1. Gheorghiade M, Abraham WT, Albert NM et al. Relationship between admission serum sodium concentration and clinical outcomes in patients hospitalized for heart failure: An analysis from the OPTIMIZE-HF registry. Eur Heart J, 2007; 28: 980-988.

2. Rossi J, Bayram M, Udelson JE et al. Improvement in hyponatremia during hospitalization for worsening heart failure is associated with improved outcomes: insights from the Acute and Chronic Therapeutic Impact of a Vasopressin Antagonist in Chronic Heart Failure (ACTIV in CHF) trial. Acute Card Care, 2007; 9: 82-86.

3. Rich MW, Beckham V, Witternberg C, Leven CL, Frredland KE, Carney RM. A multidisciplinary intervention to prevent the readmission of elderly patients with congestive heart failure. $\mathrm{N} \mathrm{Engl}$ J Med, 1995; 330: 1190-1195.

4. Lee DS, Austin PC, Rouleau JL, Liu PP, Naimark D, Tu JV. Predicting mortality among patients hospitalized for heart failure: derivation and validation for a clinical model. JAMA, 2003; 290: 2581-2587.

5. Felker GM, Leimberger JD, Coliff RM, Cuffe MS, Massie BM, Adams KF. Risk stratification after hospitalization for decompensated heart failure. J Card Fail, 2004; 10: 460-466.

6. Trullàs JC, Formiga F, Montero M et al. Paradox of obesity in heart failure: Results from the Spanish RICA Registry. Med Clin (Barc), DOI: 10.1016/j.medcli.2011.04.030.

7. Pérez-Calvo JI, Montero-Pérez-Barquero M, Camafort-Babkowski M et al. Influence of admission blood pressure on mortality in patients with acute decompensated heart failure. QJM, 2011; 104: 325-333.

8. RemmeWJ, Swedberg K. Guidelines for the diagnosis and treatment of chronic heart failure. Task Force for the diagnosis and treatment of chronic heart failure, European Society of Cardiology. Eur Heart J,2001; 22: 1527-1560.

9. Charlson ME, Pompei P, Ales KL, MacKenzie CR. A new method of classifying prognostic comorbidity in longitudinal studies: Development and validation. J Chronic Dis, 1987; 40: 373-383.

10. Mahoney FI, Barthel D. "Functional evaluation: The Barthel Index.” Maryland State Med J, 1965; 14: 56-61.

11. Martínez de la Iglesia J, Duenas-Herrero R, Onis-Vilches M, Aguado Taberne C, Albert Colomer C, Luque Luque R. Spanish language adaptation and validation of the Pfeiffer's questionnaire (SPMSQ) to detect cognitive deterioration in people over 65 years of age. Med Clin (Barc), 2001; 117: 129-134.

12. Gheorghiade M, Rossi JS, Cotts W et al. Characterization and prognostic value of persistent hyponatremia in patients with severe heart failure in the ESCAPE trial. Arch Intern Med, 2007; 167: 1998-2005.
13. Klein L, O'Connor CM, Leimberger JD, Gattis-Stough W, Pina IL, Felker GM, and OPTIME-CHF Investigators. Lower serum sodium is associated with increased short-term mortality in hospitalized patients with worsening heart failure: Results from the Outcomes of a Prospective Trial of Intravenous Milrinone for Exacerbations of Chronic Heart Failure (OPTIME-CHF) study. Circulation, 2005; 111: 2454-2460.

14. Vasan RS, Larson MG, Benjamin EJ, Evans JC, Reiss CK, Levy D. Congestive heart failure in subjects with normal versus reduced left ventricular ejection fraction: Prevalence and mortality in a population-based cohort. J Am Coll Cardiol, 1999; 33: 1948-1955 .

15. Rich MW. Epidemiology, pathophysiology, and etiology of congestive heart failure in older adults. J Am Geriatr Soc, 1997; 45: 968-974.

16. McMurray JJ, Stewart S. Epidemiology, aetiology, and prognosis of heart failure. Heart, 2000; 83: 596-602.

17. Shorr AF, Tabak YP, Johannes RS, Gupta V, Saltzberg MT, Costanzo MR. Burden of sodium abnormalities in patients hospitalized for heart failure. Congest Heart Fail, 2011; 17: 1-7.

18. Jong P, Vowinckel E, Liu PP, Gong Y, Tu JV. Prognosis and determinants of survival in patients newly hospitalized for heart failure: A population based study. Arch Intern Med, 2002; 162: 1689-1694.

19. Clarke B, Howlett J, Sapp J, Andreou P, Parkash R. The effect of comorbidity on the competing risk of sudden and nonsudden death in an ambulatory heart failure population. Can J Cardiol, 2011; 27: 254-261.

20. Wald R, Jaber BL, Price LL, Upadhyay A, Madias NE. Impact of hospital-associated hyponatremia on selected outcomes. Arch Intern Med, 2010; 170: 294-302.

21. Rochon PA, Katz JN, Morrow LA et al. Comorbid illness is associated with survival and length of hospital stay in patients with chronic disability. A prospective comparison of three comorbidity indices. Med Care, 1996; 34: 1093-1101.

22. Poses RM, McClish DK, Smith WR, Bekes C, Scott WE. Prediction of survival of critically ill patients by admission comorbidity. J Clin Epidemiol, 1996; 49: 743-747.

23. Bettari L, Mona F, Shaw LK et al. Hyponatremia and long-term outcomes in chronic heart failure: An Observational Study From the Duke Databank for Cardiovascular Diseases. J Cardiac Fail, 2012; 18: 74-81.

24. Rusinaru D, Tribouilloy C, Berry C et al. Relationship of serum sodium concentration to mortality in a wide spectrum of heart failure patients with preserved and with reduced ejection fraction: an individual patient data meta-analysis. Eur J Heart Fail, 2012; 14: 1139-1146. 